\title{
SITELLE at the CFHT
}

\section{Laurie Rousseau-Nepton ${ }^{1}$, Carmelle Robert ${ }^{1}$, Laurent Drissen ${ }^{1}$, R. Pierre Martin ${ }^{2}$, Thomas Martin ${ }^{1}$ and the SITELLE Collaboration}

${ }^{1}$ Dép. de physique, de génie physique et d'optique, Université Laval, Québec, Canada email: laurie.r-nepton.1@ulaval.ca

${ }^{2}$ Dept. of Physics \& Astronomy, University of Hawaii at Hilo, HI, USA

\begin{abstract}
SITELLE is the new imaging Fourier transform spectrograph of the Canada-FranceHawaii Telescope. It produces an impressive 4 million spectra in a single datacube in selected bandpasses from 350 to $900 \mathrm{~nm}$. Its large FOV $\left(11^{\prime} \times 11^{\prime}\right)$ and its high spatial sampling $\left(0.32^{\prime \prime} /\right.$ pixel, seeing limited) allow us to study extended objects with an unprecedented view (Drissen et al. 2014). SITELLE's first observations of nearby galaxies revealed its capabilities to conduct detailed studies of emission line regions.
\end{abstract}

Keywords. Instrum.: Imaging Spectrograph; ISM Structure; HII Regions; Abundances

\section{Summary}

Three datacubes in the spectral bands SN1 [365 - $384 \mathrm{~nm}$ ], SN2 [484 - $512 \mathrm{~nm}$ ], and SN3 [648 - $685 \mathrm{~nm}]$ were collected on the well known face-on spiral NGC 628 during SITELLE's science verification run. Data reduction was performed using ORBS (Martin 2015). More than 3500 HII regions were detected in the disk. Figure 1 shows the reconstructed spectrum of one region. The wavelength and flux calibrations are well confirmed from comparison with data from the literature.
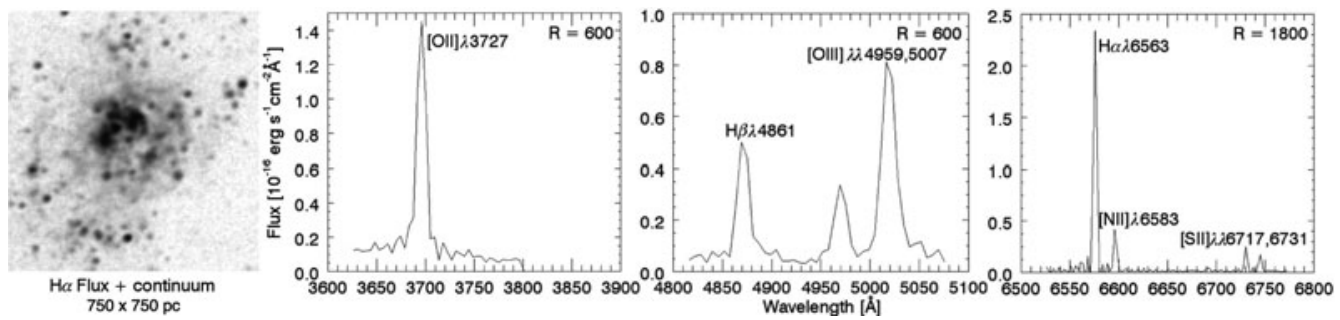

Figure 1. Details of an HII region in NGC 628, with its integrated spectrum from SITELLE.

With SITELLE, ionization structures in NGC 628 are resolved $(\sim 25 \mathrm{pc})$ and therefore, new diagnostic tools are required to better constrain the physical parameters (age, mass, abundances, ionization parameter, escaping photon fraction) of each star-forming region. The photoionization codes CLOUDY and MAPPINGS will be used to prepare a large database tailored for SITELLE in order to facilitate the comparison with different emission line ratios. A morphological classification is performed using constraints on the $\mathrm{H} \alpha$ profiles. The additional information provided by the analysis of resolved ionization morphologies and kinematics will bring new clues on the content and the physical conditions of a very large population of HII regions, along with diffuse ionized gas in disk galaxies.

\section{References}

Drissen, L., et al. 2014, AdAst, vol. 2014, id.293856

Martin, T. 2015, PhD Thesis, Université Laval, Canada. 